\title{
Sleep states detection using halfwave and Franklin transformation
}

Yash Paul ( $\square$ yashpaul1234567@gmail.com )

School of Informatics Eotvos Loránd University, Budapest, Hungary,1115

S. Fridli ( $\nabla$ fridli@inf.elte.hu )

School of Informatics Eotvos Loránd University, Budapest, Hungary, 1115

\section{Research Article}

Keywords: Sleep states, Halfwave, Faber Schauder, Franklin system, K-Nearest Neighbor, ADASYN, EEG, Blood, Nasal signal

Posted Date: May 17th, 2021

DOl: https://doi.org/10.21203/rs.3.rs-27562/v2

License: (1) This work is licensed under a Creative Commons Attribution 4.0 International License. Read Full License 


\title{
Sleep states detection using Halfwave and Franklin transformation
}

\author{
Y. Paul and S. Fridli \\ Department of Numerical Analysis, Faculty of Informatics \\ Eötvös L. University, Budapest, Hungary \\ yash@inf.elte.hu, fridli@inf.elte.hu
}

\begin{abstract}
Sleep is a physiological phenomenon and a sufficient amount of sleep is mandatory for a human for his/her health. The aim of the paper is to develop an efficient algorithm to detect the various sleep states by combining different biomedical signals. The novelty of the algorithm is that we used two piecewise linear data reduction techniques namely a new Halfwave method in time domain and Franklin transformation in frequency domain. The obtained two piecewise linear forms of signals have low complexity that still preserve the characteristics of the stages of the sleep in the signals. The components of the feature vector are generated from the parameters of the two reduced piece wise linear functions. Algorithm is tested on MIT-BIH Polysomnographic Database having more than 70 hours long term EEG, blood and nasal signals with six different sleep classes. Proposed method shows better performance in terms of average Sensitivity, Specificity, Accuracy and False Alarm Rate/hour than state of the art methods.
\end{abstract}

keywords: Sleep states, Halfwave, Faber Schauder, Franklin system, K-Nearest Neighbor, ADASYN, EEG, Blood, Nasal signal

\section{Introduction}

Sleep is a significant part of person's life and individuals used to sleep one-third of their entire life. Our research is motivated by the fact that there are large number of disorders like insomnia, breathing disorders, wake-sleep disorder sleep movement disorder found in human beings. Every sleep state has different group of neurological and physiological features. The correct identification of these features along with their states are important for diagnosis and the better treatment for such sleep disorders [1]. Sleep classification process is not a standardized one, i.e. different experts have different criteria to mark a specific period of sleep. Usually sleep scientists make classifications by using visual method to predict or decide in which state the patient is for a specific time [2]. Around $24 \%$ of the adult population have regular sleep disorders. Ohayon and Smirne [3] shown $27.6 \%$ of the Italian population have sleep problem. Gupta et al. [4] shown Indian population have $10-15 \%$ insomnia and $10 \%$ delayed sleep wave phase disorder. Worldwide this problem is increasing day by day and according to Oliver et al. [5] this problem costs around $\$ 100$ billion USD per year. According to R\&K [6] rules sleep is categorized into six categories, REM, sleep stage1, stage2, stage3, stage4 and wake state. Later on NRME2 and NRME3 are also combined resulted as just four main classes namely light sleep, Deep sleep REM and Awake state [7]. In the proposed method the implementation of Faber Schauder system with Halfwave is justified because Faber-Schauder system and Halfwave decomposition both are linear piecewise systems and the discrete version of the biomedical signals is also piecewise linear [8],[9],[10]. For accurate diagnosis of sleep phases, whole duration recordings of the selected biomedical signals needs an expert manual scoring for sleep stages using some standards, which is time consuming 
and manual scoring looks impossible for long recorded biomedical signals. Therefore, there is a need for automatic sleep phase detection to reduce cost and to increase access to diagnosis sleep stages. Usually, six EEG wave patterns are used to differentiate wake and sleep states and classify sleep stages: (1) alpha activity, (2) theta activity, (3) vertex sharp waves, (4) sleep spindles, (5) K complexes, and (6) slow wave activity [16],[11],[12],[1],[13],[14]. The main challenge to automatic sleep phase detection is heterogeneity. This means means that people around the world have different cranial structures which effect the patterns in the signal. For example 10 percent individuals don't produce alpha rhythm during stage W (wake) and 10 percent create just a constrained or limited alpha beat. This justifies the combination of other signals with EEG ( (electroencephalograph) in order to improve the results. We extend our method [15], originally developed and successfully applied for epilepsy seizure detection to the case of sleep stage classification in EEG signals.

\section{Database and channel selection}

\subsection{Database}

MIT-BIH Polysomnographic Database, collected and described by Ichimaru et al. [17],[18] at Boston's Beth Israel Hospital Sleep Laboratory. It is open source database and is freely available at (https://www.physionet.org/physiobank/database/slpdb/). It is a data collection from 16 subjects or patients whose average weight and age are $119 \mathrm{~kg}$ and 43 years respectively. The database contains over 80 hours' long data of four (C3-O1), six (C3-A1), and seven (O2-A1) channel polysomnographic recordings, each with an ECG (Electrocardiogram) signal annotated beat-by-beat, and EEG and respiration signals annotated with sleep states and apnea. Each signal is divided into 20 and 30 sec long epoch and each epoch belongs to the one of the sleep stages. The sampling rate of the measured signal is $250 \mathrm{~Hz}$ and 30 seconds duration of the EEG and other signals are labeled by associated experts. Available standard databases usually contains data of one type of signal like EEG, ECG etc. or combination of EEG, ECG. We used blood, nasal and EEG signal in proposed method and we found that this is standard and long enough database which containing blood, nasal and EEG signal, where we can test our proposed method. In our research, due to some technical problem we are not able to read 3 records out of 18 records hence, we performed our tests only on remaining 15 records from 13 different patients shown in table 4,5 and 6 .

\subsection{Channel Selection}

The channel selection is one of the most challenging tasks in sleep state detection and prediction algorithms. Considering of large number of channels will make signal processing system computationally slow. In proposed method we used data from only one channel given by the database experts. In the given database mentioned above, the experts collected the data from different biomedical signals by using only one channel and the channel selection varied from patient to patient and signal to signal (i.e no one fixed channel is used for all patient). The author of the database did not provide any more description about the channel selection process. Thus data with us is available only from one channel leaving no options for channel selection. In [15] we proposed a novel approach for channel selection for seizure detection and we planned to test the same approach here too, which is now not possible. In the future work we may test our method on the database which contains data from the different channels. 


\section{Proposed method}

The study done by Shayan et al. [16] suggests various disadvantages of the existing studies. The study motivate the researcher to do research by using some adaptive methods. Our focus is to increase the speed as well as accuracy of sleep states detection process as compared to the existing methods. Proposed method is a so called hybrid one, i.e. we utilize both time and frequency domains to extract components for the feature vector. The main idea is to use the piecewise linear function model for data reduction in time and frequency domain. Piecewise linear functions are of low complexity that still preserve the characteristics of the signal that are relevant in context of stages of the sleep. To this order we took the so called Halfwave method and modified it according to our need. The called frequency space is generated by Franklin transformation. The Franklin system an orthogonal system which is strongly related with the well-known Haar system, and its elements piecewise linear functions. Then the feature vector elements are generated from the piecewise linear models in both domains. We applied this technique for synchronized EEG, blood pressure, and respiratory signals.

5

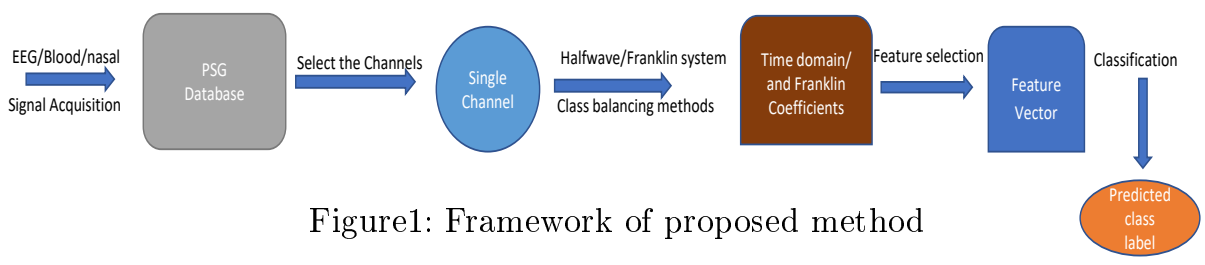

\section{Methodologies Used}

In proposed method we developed two piecewise linear time domain and frequency domain models called new Halfwave decomposition [8] and Faber-Schauder (Franklin) [9],[10] system to extract the best discriminatory features from three biomedical signals. In literature survey we have found that recent research in signal processing is surrounding around very famous transformations like wavelets, EMD, Fourier, Hilbert and Fast Fourier etc. [19]. The framework of the proposed algorithm is shown in figure 1 .

\subsection{Time domain: Halfwave method}

Traditionally, from mid of $20^{\text {th }}$ century to end of $20^{\text {th }}$ century, Halfwave was very popular method to detect epileptic activities (seizures) form the long EEG signals where the terms spikes and sharp waves also called SSWs [8] were the representative or interpretations of seizure and non seizure segments. Its main advantage is that normal and abnormal patterns of very long signals can be examined and identified easily. Different versions of half-wave methods with different criteria to define and identify Halfwave have been proposed and we asked the readers to follow these relevant publications [8],[21],[22],[23],[24],[25],[26],[27],[28] to know more about Halfwave. Our motivation differs from those above. Namely, we do not want to identify individual spikes. Instead, e.g we take a portion or full EEG and other signals, then we consider it as a piecewise linear function, and we want to simplify it by eliminating irrelevant details. To this purpose we developed new half-wave method which is simple and fast. Existing method of Hlafwave is based on three guiding principles [51] but proposed method is based on only one guiding principle out of three. First we calculate the extremal points of the original signal, drop the other values, and take 
the piecewise linear function generated by the extremal points. Since the minimum-maximum values alternate in the sequence of extremal points, the graph of the resulting piecewise-function is a type of wave form. We find that in intervals when there is a tendency of increase the decrease in the individual maxima-minima intervals are very small. They do not seem to contribute much in sleep detection process. Therefore, their inclusion in the half-wave is not necessary and can be dropped. Similar process is applied to opposite direction, i.e. when the signal shows a decreasing tendency. In the proposed method we apply only one condition of existing method [51], which is very simple, in the reduction process. After applying this condition the first time, the outcome is again a piecewise linear-wave with less extremal points, and is called first level Halfwave decomposition.The process can be repeated. It is easy to see that after some steps it will not make any further change, i.e. the next level decomposition coincides with the previous one. Then it is called final or complete Halfwave, and the previous versions are called semi-Halfwave decompositions. When we move from lower to higher levels in half-wave decomposition we loose more and more details. It is the part of the reduction problem to decide which level is the best suitable for sleep detection.

\section{Mathematical formalization of proposed Halfwave method:}

Let us suppose, that the $f:[a, b] \rightarrow \mathbb{R}$ signal is continuous on the $[a, b]$ compact interval, and suppose that $f$ has finitely many extrema on $[a, b]$. Let us first collect the extrema points in two sets:

$$
\begin{aligned}
& M_{1}:=\{x \in[a, b]: f \text { has a local maximum in } x\}, \\
& m_{1}:=\{x \in[a, b]: f \text { has a local minimum in } x\} .
\end{aligned}
$$

And let us denote the set of all extremal points by

$$
X_{1}:=M_{1} \cup m_{1}=\left\{x_{0}, x_{1}, x_{2}, \ldots, x_{n}\right\}
$$

with

$$
x_{0}<x_{1}<x_{2}<\cdots<x_{n}(n \in \mathbb{N}),
$$

such that minimum and maximum points are alternating.

In the $k$ th step of the proposed algorithm $(k=1,2,3, \ldots)$ we delete some extremal points from $M_{k}, m_{k}$ and $X_{k}$, and we will keep only the important extremal points to get the new sets $M_{k+1}, m_{k+1}$ and $X_{k+1}=M_{k+1} \cup m_{k+1}$. The algorithm will converge, i.e.

$$
\exists K \in \mathbb{N}: \forall k \geq K: M_{k}=M_{K}, m_{k}=M_{K},
$$

but we will stop at a suitable iteration number $k^{*}$.

One step of the proposed algorithm (deleting unwanted extrema) can be formulated as follows. We start with $M_{k}, m_{k}$ and $X_{k}=M_{k} \cup m_{k}$, with the elements of $X_{k}$ indexed in ascending order, as above. Now define

$$
y_{i}=f\left(x_{i}\right)(i=0, \ldots, n)
$$

the extremal function values,

$$
\Delta_{i}:=y_{i+1}-y_{i}(i=0, \ldots, n-1),
$$

the differences between two consecutive extreme values (a minimum and a maximum), and

$$
\begin{aligned}
D:= & \{i \in \mathbb{N}: 1 \leq i<n-1 \\
& \left.\left|\Delta_{i}\right| \leq\left|\Delta_{i+1}\right| \wedge\left|\Delta_{i}\right| \leq\left|\Delta_{i-1}\right|\right\} \cup\{n\}
\end{aligned}
$$


the set of indexes of segments with not significant difference (less than both neighboring segments). To formulate the set of important extremal points we will use the strictly increasing index function

defined by $\nu(0)=0$ and

$$
\nu:\{0,1, \ldots, N\} \rightarrow\{0,1, \ldots, n\} .
$$

$$
\nu(j+1)=\nu(j)+2 d+1(j=0,1, \ldots N-1 ; d \in \mathbb{N}),
$$

such that

$$
\begin{gathered}
\nu(j)+2 d+1 \notin D, \\
\nu(j)+2 \delta+1 \in D(\delta=0,1, \ldots, d-1) .
\end{gathered}
$$

(It will turn out that $\nu(N)=n$ ). And then the set of important extremal points can be written as

$$
X_{k+1}:=\left\{x_{\nu(j)} \in X_{k}: j=0,1, \ldots, N\right\} \subset X_{k} .
$$

As a consequence of the definition the values in $X_{k+1}$ are alternatively minimum and maximum points. Furthermore we can also formulate

$$
\begin{aligned}
M_{k+1} & =X_{k+1} \cap M_{k}, \\
m_{k+1} & =X_{k+1} \cap m_{k}
\end{aligned}
$$

the sets of the new maximum and minimum values.

After the completion of first level, we will repeat the same procedure for the outcome $\left(M_{k+1}, m_{k+1}\right.$ and $\left.X_{k+1}\right)$ of the first level to get signal at next level (level 2) and so on until required level is found.

\subsection{Frequency domain: Franklin transform}

The frequency domain part in our hybrid classification is an orthogonal projection using a proper orthogonal system. As we know our model space for the EEG and other signals is the family of continuous piecewise continuous functions. In order to perform an orthogonal projection, reduction in the frequency domain, that complies with our model we need an orthogonal system that consists of continuous piecewise continuous functions. This guarantees that the subspace spanned by the elements of the system is a subspace of our model space. The combination of these requirements motivate us to consider the Franklin system as a natural choice.

Franklin system: The first example for an orthogonal basis in the space of continuous functions Franklin system is constructed from the well known Haar system [29]. Integral Haar functions is called Faber-Schauder system [9],[10]. Franklin functions are piecewise linear functions. They follow the structure of the Haar system: the nodes are $\frac{k}{2^{n}}$ for the first $2^{n}$ elements. A Franklin system is an orthogonal system of basis which is derived from Faber Schauder system of basis by applying Gram-Schmidt orthogonal procedure [31],[32],[33] on Faber Schauder system. The Franklin system has the same linear span as that of Faber Schauder systems and this span is dense in $\mathrm{C}([0,1])$, hence $L^{2}([0,1])$ consists of continuous piecewise linear function.

\section{$5 \quad$ Features Extraction and classification}

\subsection{Feature Extraction}

In proposed method a hybrid approach is used to construct the final feature vector. Feature vector is constructed from the three biomedical signals. The sampling rate of the measured signal 
is $250 \mathrm{~Hz}$ and 30 seconds duration of the EEG and other signals are labeled by associated experts to categorize different states of the sleep.

Feature extraction in time domain : Halfwave method Biomedical experts annotated the selected signals, where each and every segment of $30 \mathrm{sec}$ long is marked with a specific sleep state. Many other studies and methods cited here worked with a window size of $30 \mathrm{sec}(7500$ sample points) therefore, we also using the the same window size. By using new Halfwave method we decomposed the signal up to level 2 only. The reason to use Halfwave up to level 2 is that when we go deeper in the level in blood, nasal and EEG signals, the signals become so simple that in some of the $30 \mathrm{sec}$ long segments have only 1 extrema point from which we cannot extract the desired features. In selected signals this type of situation occurs in very early levels because the nature of the signals in case of the sleep states (slow activities less peaky) quite different than seizure states (fast and peaky activities). Therefore we could not use the level as proposed in [15] because the nature of signal is different. Thus, this is the advantage of Halfwave method that we can customize the method based on the problems in hands. The time domain features used here are: total number of extrema points, mean of the absolute values of the extrema points, maximum of the absolute values of the extrema points, maximum, of the absolute values of the slopes, mean of absolute values of slopes and the last feature sum of the square of extrema points which related to the energy of the signal are extracted. The reason of choosing these features is that these are the simple and very common statistical features. Moreover, various studies and surveys [15],[16],[19] also summarize that these are the best and are frequently used features in time domain for the analysis of the different biomedical signals. In practice we extracted number of statistical features in time domain and then an analysis has been made on these features by using histograms [15]. We found that the selected 6 time domain features are the best discriminatory features and their combination with selected Franklin coefficients gives better results than any other combination as shown in table 1.

Feature extraction in frequency domain : Franklin Transformation In frequency domain the segmentation of the signal by using rectangular window is little bit tricky. If we take window size $30 \mathrm{sec}$ long ( 5700 samples) then there is no linearity holds between any dyadic rationals of the form $K 2^{-8}(k=0, \ldots, 256)$. Thus, 1 segment of $30 \mathrm{sec}$ long with 7500 sample points, needs to be re-sampled to 256 samples points so that linearity must hold between any dyadic rationals of the form $K 2^{-8}(k=0, \ldots, 256)$. But the problem with this drastic reduction from 7500 to 256 samples is that, we may loose some important information. At this point of time we are not able to give the solid justification for such drastic reduction from 7500 to 256 samples except the linearity must hold between any dyadic rationals of the form $K 2^{-8}(k=0, \ldots, 256)$, but if we look at the comparisons of results with state of the art methods, then we believe that proposed algorithm can be used in this way too. We are further working to enhance the proposed algorithm and we hope in the future work we will have more improved results with solid justification the way, the method we used (drastic reduction) here.We re-sampled the signal by using resample() function of the matlab which is $y=\operatorname{resample}(x, p, q)$ where the function resamples the sequence $x$ at $p / q$ times the original sample rate. The length of the result $y$ is $p / q$ times the length of $\mathrm{x}$. After applying the Franklin transformation on the selected segment we will get 256 coefficients. To find the best features out of 256 coefficients we made some combinations (table1) with selected time domain features and it is found that first 8 coefficients are the best discriminatory features.

Final feature vector construction From three biomedical signals (EEG, blood, nasal) we have three feature vectors where each feature vector is constructed by merging the 6 time 
domain and first 8 frequency domain features and this feature vector has a total of 14 features. Halfwave reduction technique is applied on original nasal and blood signal and then same set of 6 statistical features are extracted from each and every $30 \mathrm{sec}$ long segment. Let V1, V2, $\mathrm{V} 3$ and are the feature vectors extracted from EEG, blood and nasal respectively. Let $\mathrm{F}$ be the final feature vector used for classification, therefore, then $F=[\mathrm{V} 1 \mathrm{~V} 2 \mathrm{~V} 3]$. Thus the final feature vector used for classification here has 42 features. In this way the features from three biomedical signals are merged. In the table $1,6 \mathrm{~T}$ means, six best time domain features and $8 \mathrm{~F}=$ First Eight Franklin coefficients. Final results with different set of classes are shown in table number 2, 3, 4,5 and 6 where training and Testing data is taken in the ratio of $60: 40$ respectively.

\subsection{Classification}

After the feature vector construction we applied the K-Nearest Neighbor algorithm (KNN) for classification. We found from the various literature surveys that in some applications KNN performs better in this task than other often used classification algorithms like Support Vector Machine (SVM), Artificial Neural Network (ANN) etc. In this regard we agrees with the conclusions of other authors mentioned in different studies. We note that the sets of sleep stages in the databases are not balanced. This means that the numbers of tuples of different classes are significantly different. Therefore, before applying the classifier, the class imbalance problem needs to be addressed otherwise results would be biased. In the proposed method we used an advanced form of Synthetic Minority Over-Sampling Technique (SMOTE) [34] to address the issue of class imbalance. It is called Adaptive Synthetic Sampling Approach for Imbalanced Learning [35].

Table 1: Feature selection using different combinations of the signals

\begin{tabular}{|c|c|c|c|c|c|c|}
\hline Signal & Class-pair & Features & Train\&Test & Sensitivity $(\%)$ & Specificity $(\%)$ & Accuracy $(\%)$ \\
\hline Blood & 4 & $6 T+16 F$ & $60-40$ & 91.02 & 97 & 91 \\
\hline Blood & 4 & $6 T+16 F$ & $80-20$ & 96.11 & 98.70 & 96.09 \\
\hline Blood & 4 & $6 T+8 F$ & $80-20$ & 96.03 & 98.67 & 96.02 \\
\hline Blood & 4 & $6 T+8 F$ & $60-40$ & 90.64 & 96.86 & 90.60 \\
\hline Resp & 4 & $6 T+8 F$ & $60-40$ & 91.11 & 97.05 & 91.15 \\
\hline Resp & 4 & $6 T+8 F$ & $80-20$ & 96.37 & 98.79 & 96.38 \\
\hline Resp & 4 & $6 T+16 F$ & $60-40$ & 90.87 & 96.98 & 90.97 \\
\hline Resp & 4 & $6 T+16 F$ & $80-20$ & 96.15 & 98.72 & 96.17 \\
\hline EEG & 4 & $6 T+8 F$ & $80-20$ & 95.63 & 98.58 & 95.63 \\
\hline EEG & 4 & $6 T+8 F$ & $60-40$ & 90 & 96.7 & 90 \\
\hline EEG & 4 & $6 T+16 F$ & $80-20$ & 90.64 & 96.86 & 90.60 \\
\hline Blood Resp EEG & 4 & $6 \mathrm{~T}+8 \mathrm{~F}$ & $60-40$ & 93.28 & 97.76 & 93.28 \\
\hline Blood Resp EEG & 4 & $6 \mathrm{~T}+8 \mathrm{~F}$ & $80-20$ & 97.28 & 99.09 & 97.27 \\
\hline Blood Resp EEG & 4 & $6 T+16 F$ & $60-40$ & 92.82 & 97.60 & 92.84 \\
\hline Blood Resp EEG & 4 & $6 T+16 F$ & $80-20$ & 96.92 & 98.97 & 96.93 \\
\hline Blood Resp EEG & 4 & $6 T+32 F(114)$ & $80-20$ & 96.51 & 98.83 & 96.50 \\
\hline Blood Resp & 4 & $6 T+16 F(44)$ & $80-20$ & 96.71 & 98.99 & 96.77 \\
\hline Blood Resp & 4 & $6 T+16 F(44)$ & $60-40$ & 92.37 & 97.45 & 92.37 \\
\hline
\end{tabular}

\section{Related Work}

In the literature survey we studied number of sleep states detection techniques and we found that recent research is focusing on dynamic parameters like correlation dimension, Lyponov exponent, approximate entropy, mean, energy of the signal, slopes, etc. to extract comprehensive information from non linear signals like EEG, blood and respiratory [36]. Originally the Halfwave was used in seizure detection but new Halfwave method proposed by us can be used with Franklin 
transformation (a hybrid approach) [15] to detect epileptic seizures and sleep states classifications in an efficient way by using different biomedical signals. We believe that this method with slight modification in the parameters if needed can be useful to solve many problems in biomedical field in an efficient way. Dihong et al. [37] used three biomedical signals like using EEG, EOG and EMG and on an average, accuracy of $81.2 \%$ and a Cohen's Kappa coefficient of 0.722 are obtained under leave-one-subject-out cross validation. Nicola et al. [38] proposed single channel automated detection of sleep states using EEG signals. Time domain and frequency domain features are classified for four and two sleep stages separately with $90.81 \% 83.2 \%$ respectively. They achieved an overall accuracy of $86.7 \%$. Tripathy et al. [39] they used dispersion entropy and the variance features from the different bands of EEG signal. The RR-time series features and the EEG features feed to the deep neural network (DNN) to carry out the classification of sleep stages. They achieved an average accuracy of $85.51 \%, 94.03 \%$ and $95.71 \%$ for the classification of 'sleep vs wake', 'light sleep vs deep sleep' and 'rapid eye movement (REM) vs non-rapid eye movement (NREM)' sleep stages. Silverira et al. [40] proposed a single channel method where EEG signal is decomposed using wavelet transform. The features such as kurtosis, skewness and variance of the wavelet coefficients are classified using random forest classifier and they obtained an over all accuracy for 2 to 6 classes is $90 \%$. Budak et al. [41] they proposed new method to detect driver drowsiness.They decompose the signal using Q-factor wavelet transform in sub-bands. The Spectrogram images of the obtained sub-bands and statistical features like standard deviation of instanious frequencies are calculated. Features are classified by long-short term memory (LSTM) for classification. They obtained an over all accuracy of 94.31 for awake and drowsy (S1) state. Taran et al. [42] used Hermite functions as basis functions and the Hermite coefficients are used as features to classify alertness and drowsiness states. With ELM (Extreme Learning Method) their detection rate for alert and drowsiness are $95.45 \%$ and $87.92 \%$. The over all accuracy was $92.28 \%$. A subject specific approach [43] where 12 features are extracted by three methods namely, the heart rate variability (HRV), detrended fluctuation analysis (DFA) and windowed DFA (WDFA). They reported an average accuracy of 79.99 and kappa coefficient 0.43 . Another subject specific approach is mentioned in [44] where average accuracy are using EEG is $76 \%$ and using ECG signals is $75 \%$.

\section{Experimental Results and Discussions}

Tests were performed on 15 records (out of 18 records) and total duration of selected 15 long term EEG, blood and nasal signals with six different sleep classes is more than 70 hours. First we have optimized our method then we have carried out comparison tests. It turned out that the proposed method shows better performance in terms of an average accuracy, sensitivity, specificity, and False alarm rate/ hr than state of the art methods. The performance is evaluated by the following metrics. Sensitivity $=\frac{\mathrm{TP}}{\mathrm{TP}+\mathrm{FN}} \times 100$

$$
\begin{aligned}
& \text { Specificity }=\frac{\mathrm{TN}}{\mathrm{TN}+\mathrm{FP}} \times 100 \\
& \text { Accuracy }=\frac{\mathrm{TP}+\mathrm{TN}}{\mathrm{TN}+\mathrm{FP}+\mathrm{TP}+\mathrm{FN}} \times 100
\end{aligned}
$$

False alarms per hour $(\mathrm{Fph})=$ the total number of false detection divided by the length of the test data in hour.

where $\mathrm{TP}$ is true positive, $\mathrm{TN}$ is true negative, $\mathrm{FN}$ is false negative.

The various results obtained are shown in table $1,2,3,4,5$ and table 6 . The table 1 helps us to choose best set of discriminatory features among large number of features and we found that the combination with bold text in the table 1 can be consider as best combination to detect various sleep states. Table 2, table 3 shows the comparison with state of the art methods with same and different database respectively and it shows that our algorithm performed better than 
Table 2: Comparison of results with state-of-the art algorithms tested on the same database

\begin{tabular}{|c|c|c|c|c|c|}
\hline Author and year & Number of records & features & Classes used & Classifier used & Average Accuracy( \%) \\
\hline $\begin{array}{lr}\text { Redmond } & \text { and } \\
\text { Heneghan } & {[44] \text {, }} \\
2003 & \\
\end{array}$ & 17 & $\begin{array}{l}\text { HRV features and } \\
\text { EEG Features }\end{array}$ & $\begin{array}{l}\text { Light sleep vs deep } \\
\text { sleep }\end{array}$ & QDA & 89 \\
\hline $\begin{array}{l}\text { Adnane et al. } \\
{[43], 2012}\end{array}$ & 17 & HRV, DFA and WDFA & Sleep vs wake & SVM & 79.99 \\
\hline $\begin{array}{l}\text { Hayet and Slim } \\
{[45], 2012}\end{array}$ & 09 & $\begin{array}{l}\text { RR-time series and } \\
\text { HRV features }\end{array}$ & Sleep vs wake & ELM & 83.59 \\
\hline $\begin{array}{l}\text { Werteni et al. } \\
{[46], 2015}\end{array}$ & 17 & HRV & Sleep vs wake REM & SVM & 56.81 \\
\hline $\begin{array}{l}\text { R.K Tripathi et } \\
\text { al. [39], May, } \\
2018\end{array}$ & 17 & $\begin{array}{l}\text { Dispersion entropy and } \\
\text { variance }\end{array}$ & $\begin{array}{l}\text { wake, light sleep, deep } \\
\text { sleep, REM }\end{array}$ & Neural network & $85.51,94.095 .71$ \\
\hline $\begin{array}{l}\text { Taran et al. [42], } \\
2018\end{array}$ & 16 & Hermite coefficients & $\begin{array}{l}\text { alert(w) and drowsi- } \\
\text { ness(s1) }\end{array}$ & ELM & 92.28 \\
\hline $\begin{array}{l}\text { Budak et al. } \\
\text { [41], } 2019\end{array}$ & 16 & $\begin{array}{l}\text { spectrogram images } \\
\text { and instanious fre- } \\
\text { quencies }\end{array}$ & alert and drowsiness & LSTM & $94.31 \%$ \\
\hline $\begin{array}{l}\text { Panfeng et al. } \\
{[47], 2019}\end{array}$ & 06 & statistical features & $\begin{array}{l}\text { NREM (s1-s4), REM, } \\
\text { Wake }\end{array}$ & W-SVM & 85.29 \\
\hline $\begin{array}{l}\text { Junming at el. } \\
{[48], 2020}\end{array}$ & 18 & $\begin{array}{l}\text { Hilbert Huang coeffi- } \\
\text { cients }\end{array}$ & REM,REM, wake & $\mathrm{CNN}$ & 87.60 .81 \\
\hline $\begin{array}{l}\text { Proposed } \\
\text { method }\end{array}$ & 15 & $\begin{array}{l}\text { time domain and } \\
\text { Franklin coefficients }\end{array}$ & Wake, Sleep(all), REM & KNN & 96.9,93.94, 93.84 \\
\hline
\end{tabular}

other state of the art methods.Our method is patient specific and the state of the methods used for comparisons are also patient specific, therefore it justifies the comparisons. Table $4,5,6$ shows classification results for randomly selected 2,4 , and 6 classes respectively.

\section{Conclusion and future work}

A novel hybrid approach of two piecewise linear models has been developed to extract the features from the biomedical signals. The main idea behind the two piecewise linear models is to morph the signals in such a way that signal should become simple and smooth but at the same it must retain the important characteristics of the sleep states in it. Different time domain and frequency domain features are extracted, and these features are combined to construct final feature vector. Features are classified by using KNN classifier on long data of CHB-MIT polysomnography database. Proposed algorithm achieved an average sensitivity, specificity, accuracy and false alarm rate of $98.35 \%$ and $97.32 \%, 96.96 \%, 0.029$ respectively for two randomly picked classes, $96.62 \%$ and $97.10 \%, 93.94 \%, 0.030$ for randomly picked any 4 classes, $96.13 \%$ and $98.33 \%, 93.84 \%$, 0.016 for all six classes, which is higher so far than state of the art methods. In future algorithm will be tested on very long data of different databases. In this algorithm we have used three biomedical signals which may slow down the speed of the system instead of two or less signal being used. Therefore in near future we will try to use only two or less signals with different set of features from different signals so that further results can be improved in an more efficient way. In future, we plan to use EEG and blood signal where Franklin system may be used on EEG and some time domain features can be extracted from blood signal.

\section{Funding and Acknowledgments}

EFOP-3.6.3-VEKOP-16-2017-00001: Talent Management in Autonomous Vehicle Control Technologies - The Project is supported by the Hungarian Government and co-financed by the European Social Fund. 
Table 3: Comparison of results with other algorithms tested on the different database

\begin{tabular}{|c|c|c|c|c|}
\hline Author and year & features & Classes used & Classifier used & Average Accuracy(\%) \\
\hline Prucnal et al. [49] & $\begin{array}{l}\text { EMD and wavelet } \\
\text { based features }\end{array}$ & $\begin{array}{l}\text { Five class (wake, S1, } \\
\text { S2, deep sleep, REM) }\end{array}$ & Neural Network & $\begin{array}{ll}74.2 \quad \text { (using } & \text { DWT } \\
\text { features), } 57.6 & \text { (using } \\
\text { EMD features) }\end{array}$ \\
\hline Hasan et al. [50] & $\begin{array}{l}\text { Ensemble EMD based } \\
\text { features }\end{array}$ & $\begin{array}{l}\text { Six classes (wake, S1, } \\
\text { S2, S3, S4, REM) }\end{array}$ & RUSBoost & $\begin{array}{l}42.05 \text { (S1), } 79.51 \text { (S2), } \\
86.61 \text { (S3), } 48.09 \text { (S4), } \\
95.16 \text { (wake), } 80.50 \\
\text { (REM sleep) }\end{array}$ \\
\hline Da Silveira et al. [40] & $\begin{array}{l}\text { DWT and statistical } \\
\text { features }\end{array}$ & $\begin{array}{l}\text { (wake, S1, S2, S3, S4, } \\
\text { REM) }\end{array}$ & Random forest & $\begin{array}{l}5.80 \text { (S1), } 87.70 \text { (S2), } \\
51.50 \text { (S3), } 68.00 \text { (S4), } \\
99.3 \text { (wake), } 68.80 \\
\text { (REM sleep) }\end{array}$ \\
\hline $\begin{array}{l}\text { R.K Tripathi et al. } \\
{[14], \text { May, } 2018}\end{array}$ & HRV features & (NREM, REM) & DNN & 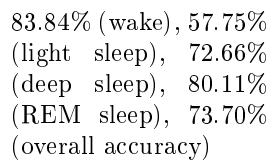 \\
\hline Proposed method & $\begin{array}{l}\text { time domain and } \\
\text { Franklin coefficients }\end{array}$ & $\begin{array}{l}\text { Wake, Sleep (all), } \\
\text { REM }\end{array}$ & KNN & $96.9,93.94,93.84$ \\
\hline
\end{tabular}

\section{References}

[1] Herrera, L. J. et. al. Combination of heterogeneous EEG feature extraction methods and stacked sequential learning for sleep stage classification, International Journal of Neural Systems 23(03), pp. 1-201350012 (2013).

[2] Norman, R. and Robert, G. et. al. Interobserver Agreement Among Sleep Scorers From Different Centers in a Large Dataset, Sleep, vol. 23, no. 7 (2000), pp. 1-8, https://doi.org/10.1093/sleep/23.7.1e. November (2000)

[3] Ohayon, M. and Smirne, S. Prevalence and consequences of insomnia disorders in the general population of italy, Sleep Medicine, vol. 3, no. 2, pp. 115-20, 2002. [Online]. http://www.sciencedirect.com/science/article/pii/S1389945701001587.

[4] Gupta, R. et. al. Clinical practice guidelines for sleep disorders, Indian J Psychiatry ;59, Suppl S1:116-38, vol. 59, pp. 116-38, 2017.

[5] Faust, O., et. al. A review of automated sleep stage scoring based on physiological signals for the new millennia, Computer Methods and Programs in Biomedicine, vol. 176, pp. 19-30, Sep., 2019.

[6] Rechtschaffen, A. and Kales, A. A manual of standardized terminology, techniques and scoring system for sleep stages of human subjects,Public Health Service, US Government Printing Office, Washington DC, 071968.

[7] Iber, C., et. al. The aasm manual for the scoring of sleep and associated events: Rules, terminology and technical specifications, Westchester, IL: American Academy of Sleep. Medicine, 012007. 
Table 4: Results of proposed algorithm with 2 randomly selected classes

\begin{tabular}{ccccc}
\hline Patient number & Sensitivity $(\%)$ & Specificity $(\%)$ & Accuracy $(\%)$ & Falsealarmrate $/ \mathrm{hr}$ \\
\hline 01a & 100 & 96.30 & 96.61 & 0.037 \\
\hline 01b & 100 & 99.75 & 99.75 & 0.025 \\
\hline 2a & 96.53 & 97.69 & 97.21 & 0.0231 \\
\hline $2 \mathrm{~b}$ & 100 & 96.30 & 96.69 & 0.037 \\
\hline 03 & 100 & 97 & 97.10 & 0.035 \\
\hline 04 & 100 & 95.45 & 96.01 & 0.045 \\
\hline 14 & 100 & 96 & 97.02 & 0.030 \\
\hline 16 & 97.01 & 92.16 & 93.95 & 0.078 \\
\hline 37 & 100 & 97.46 & 97.56 & 0.025 \\
\hline 48 & 94.31 & 95.61 & 94.21 & 0.065 \\
\hline 59 & 96.53 & 97.69 & 97.21 & 0.0231 \\
\hline 60 & 95.52 & 100 & 96.08 & 0.01 \\
\hline 61 & 96.49 & 99.83 & 96.45 & 0.005 \\
\hline 66 & 100 & 98.86 & 98.90 & 0.011 \\
\hline $66 \mathrm{x}$ & 100 & 99.72 & 99.73 & 0.002 \\
\hline Avg & $\mathbf{9 8 . 3 5}$ & $\mathbf{9 7 . 3 2}$ & $\mathbf{9 6 . 9 6}$ & $\mathbf{0 . 0 2 9}$
\end{tabular}

[8] Gotman, J. and Gloor, P. Automatic recognition and quantification of inter-ictal epileptic activity in the human scalp EEG, Electroencephalography and Clinical Neurophysiology, vol. 41, pp. 513-529, 1976.

[9] Faber, G. Über die Orthogonal Funktionen des herrn Haar, Deutsche Math.-Ver (in German), vol. 19, pp. 104-112, 1910.

[10] Golubov, B. Faber-schauder system, in Hazewinkel, Michiel, Encyclopedia of Mathematics, Springer Science+Business Media B.V. / Kluwer Academic Publishers, ISBN 978-1-55608010-4, p. 317-320, 1994.

[11] http://www.ninds. nih.gov/. (15 Sept 2014).

[12] Acharya, U., et. al. it Non-linear analysis of eeg signals at various sleep stages, Computer methods and programs in biomedicine 80 (2005) 37-45. doi:10.1016/j.cmpb.2005.06.011.

[13] Garces, A. Orosco, L. et. al. Automatic detection of drowsiness in EEG records based on multimodal analysis, Med. Eng. Phys 36. PP. 244-249(2014) .

[14] Tripathy, R. Application of intrinsic band function technique for automated detection of sleep apnea using HRV and EDR signals, Biocybernetics and Biomedical Engineering 38(1) (2018) 136-144. doi:https://doi.org/10.1016/j.bbe.2017.11.003.

[15] Paul, Y. and Fridli, S. Epileptic Seizure Detection Using Piecewise Linear Reduction. Lecture Notes in Computer Science, Springer, Cham., vol 12014. (2020), 364-371, https://doi.org/10.1007/978-3-030-45096-0_45

[16] Motamedi-Fakhr, et. al. Signal processing techniques applied to human sleep EEG signals - a review, Biomedical Signal Processing and Control, 10, 21-33. (doi:10.1016/j.bspc.2013.12.003). 
Table 5: Results of proposed algorithm with 4 randomly selected classes

\begin{tabular}{ccccc}
\hline Patient number & Sensitivity(\%) & Specificity(\%) & Accuracy(\%) & Falsealarmrate $/ \mathrm{hr}$ \\
\hline $01 \mathrm{a}$ & 100 & 95.41 & 95.89 & 0.045 \\
\hline $01 \mathrm{~b}$ & 97.15 & 98.31 & 94.45 & 0.0169 \\
\hline 2a & 96.51 & 98.64 & 95.89 & 0.0136 \\
\hline 2b & 100 & 95.41 & 95.89 & 0.041 \\
\hline 03 & 97.12 & 97.65 & 94 & 0.022 \\
\hline 04 & 93.71 & 93.85 & 91 & 0.0604 \\
\hline 14 & 95.90 & 97.14 & 93 & 0.028 \\
\hline 16 & 97.10 & 97.64 & 93.64 & 0.023 \\
\hline 37 & 98.44 & 98.57 & 94.70 & 0.014 \\
\hline 48 & 93.81 & 93.95 & 91 & 0.0605 \\
\hline 59 & 96.51 & 98.64 & 95.89 & 0.0236 \\
\hline 60 & 95.62 & 97.07 & 92.23 & 0.0293 \\
\hline 61 & 96.84 & 98.32 & 94.48 & 0.0168 \\
\hline 66 & 93.55 & 96.87 & 91.53 & 0.0131 \\
\hline $66 \mathrm{x}$ & 96.47 & 98.12 & 94.81 & 0.0188 \\
\hline Avg & $\mathbf{9 6 . 6 2}$ & $\mathbf{9 7 . 1 0}$ & $\mathbf{9 3 . 9 4}$ & $\mathbf{0 . 0 3 0}$
\end{tabular}

[17] Ichimaru, Y., Moody, G. Development of the polysomnographic database on cd-rom, PCN Psychiatry and clinical neurosciences 53 (1999) 175-177. doi:https://doi.org/10.1046/j.14401819.1999.00527.x. (1999).

[18] Goldberger, A., et. al. Physiobank, physiotoolkit, and physionet:Components of a new research resource for complex physiologic signals 101 (23) (2000) 215-220. doi:10.1161/01.CIR. 101.23.e215.

[19] Paul, Y. Various epileptic seizure detection techniques using biomedical signals: a review, Brain Inf. 5 (6) (2018) 429-436. doi:https: //doi.org/10.1186/s40708-018-0084-z.

[20] Jasper, H. and Kershman, J. Electroencephalographic classification of the epilepsy, Archives of Neurology and Psychiatry 45 (6) (1941) 903-943.

[21] Chatrian, G. et. al. Glossary of terms most commonly used by clinical etectroencephalographers, Electroencephalography and Clinical Neurophysiology 37 (1974) 538-548.

[22] Kooi, K. Voltage-time characteristics of spikes and other rapid electroencephalographic transients: semantic and morphological considerations., Neurology (Minneap.) 16 (1) (1996) 5966. doi:10.1212/wnl.16.1.59.

[23] Walter, D.O., Muller, H. F. and Jell, R. M. Semiautomatic quantification of sharpness of EEG phenomenon, IEEE Trans. biomed. Engng BME 20 (1973) 53-54.

[24] Saltzberg, B., Lustick, L. S., and Heath, R. G. Detection of focal depth spiking in the scalp EEG of monkeys, Electroenceph. clin. Neurophysiol 31(1971) 327-333.

[25] Da Silva, F. L., et. al. Automatic detection and localization of epileptic foci, Electroencephalography and Clinical Neurophysiology 43 (1) (1977) 1-13. doi:https://doi.org/10.1016/0013-4694(77)355 90189-4. 
Table 6: Results of proposed algorithm with 5 and six randomly selected classes

\begin{tabular}{|c|c|c|c|c|}
\hline Patient number & Sensitivity $(\%)$ & Specificity $(\%)$ & Accuracy $(\%)$ & Falsealarmrate/hr \\
\hline $01 \mathrm{a}$ & 97.09 & 98.77 & 97.09 & 0.0123 \\
\hline $01 \mathrm{~b}$ & 98.05 & 98.68 & 93.31 & 0.0132 \\
\hline $2 \mathrm{a}$ & 93.69 & 98.11 & 98.78 & 0.0122 \\
\hline $2 b$ & 96.69 & 97.92 & 93.50 & 0.020 \\
\hline 03 & 96.78 & 98.59 & 94.11 & 0.014 \\
\hline 04 & 94.25 & 97.40 & 90.59 & 0.026 \\
\hline 14 & 97.34 & 98.85 & 94.67 & 0.0115 \\
\hline 16 & 97.67 & 98.78 & 95.39 & 0.00122 \\
\hline 37 & $N A$ & $N A$ & $N A$ & $N A$ \\
\hline 48 & 94.25 & 97.40 & 90.59 & 0.0260 \\
\hline 59 & 94.79 & 98.58 & 93.10 & 0.0188 \\
\hline 60 & $N A$ & $N A$ & $N A$ & $N A$ \\
\hline 61 & 96.94 & 98.63 & 94.19 & 0.0137 \\
\hline 66 & $N A$ & $N A$ & $N A$ & $N A$ \\
\hline $66 x$ & $N A$ & $N A$ & $N A$ & $N A$ \\
\hline Avg & 96.13 & 98.33 & 93.84 & 0.016 \\
\hline
\end{tabular}

[26] Gotman, J., Gloor, P. and Ray, W. A quantitative comparison of traditional readings of the EEG and interpretation of computer-extracted features in patients with supratentorial brain lesions, Electroencephalography and clinical neurophysiology 38 (6) (1975) 623-639. doi:https://doi.org/10.1016/0013-4694(75)90163-7.

[27] Jean, G., et. al., Clinical applications of spectral analysis and extraction of features from electroencephalograms with slow waves in adult patients, Electroenceph, clin. Neurophysio 35 (3) (1973) 225-235. doi:https://doi.org/10.1016/0013-4694(73)90233-2.

[28] Gevins, A., et. al. Mapping cognitive brain function with modern highresolution electroencephalography, Trends in Neurosciences 18 (10) (1995) 429-436. doi:https://doi.org/10.1016/0166-2236(95)94489-R.

[29] Haar, A. and Zur Theorie Der Orthogonalen Funktionensysteme, Mathematische Annalen 69 (3) (1910) 331-371.

[30] Lindenstrauss, J., and Tzafriri, L. Classical banach spaces i, sequence spaces, Ergebnisse der Mathematik und ihrer Grenzgebiete, Springer-Verlag,ISBN 3-540-08072-4 (1977) 3.

[31] Ciesielski, Z. Properties of the Orthonormal Franklin System, Encyclopedia of Mathematics 23 (1963) 141-157. doi:http://eudml.org/doc/217068.

[32] Golubov, B. Franklin System (Originator), www.encyclopedia.com.

[33] Franklin, P. A set of continuous orthogonal functions, Math. Ann 100 (1928) 522-529.

[34] Chawla, N., Bowyer, K., and Kegelmeyer, W. Smote: synthetic minority over-sampling technique, Journal of Artificial Intelligence Research 16 (2002) 321-357. doi:10.1613/jair.953.

[35] He, H., Bai, Y., Garcia, E., Li, S. Adasyn: Adaptive synthetic sampling approach for imbalanced learning, Proceedings of the International Joint Conference on Neural Networks (2008) 1322-1328doi:10.1109/IJCNN.2008.4633969. 
[36] Liu, Z., Sun, J., Zhang, Y., and Rolfe, P. Sleep staging from the EEG signal using multi-domain feature extraction, Biomedical Signal Processing and Control 30 (2016) 86-97. doi:10.1016/j.bspc.2016.06.006.

[37] Jiang, D., MA, Y., Wang, Y. Sleep stage classification using covariance features of multichannel physiological signals on riemannian manifolds, Computer Methods and Programs in Biomedicine 178 (2019) 186-190. doi:10.1016/j.cmpb.2019.06.008.

[38] Michielli, N., Acharya, U.R. and Molinari, F. Cascaded lstm recurrent neural network for automated sleep stage classification using single-channel EEG signals, Computers in Biology and Science 106 (3) (2019) 71-78.

[39] Tripathy, R. and Acharya, U.R. Use of features from R-time series and EEG signals for automated classification of sleep stages in deep neural network framework, Biocybernetics and Biomedical Engineering 38 (4) (2018) 890-902.

[40] Da Silveira, T., Kozakevicius, A. and Rodrigues, C. Single channel eeg sleep stage classification based on a streamlined set of statistical features in wavelet domain, Med Biol Eng Comput 55(2) (2017) 343-52.

[41] Budak, U. et. al. An effective hybrid model for EEG-based drowsiness detection, IEEE Sensors Journal 19 (17) (2019) 7624-7631. doi:10.1109/JSEN.2019.2917850.

[42] Taran, S. and Bajaj, V. Drowsiness detection using adaptive Hermite decomposition and extreme learning machine for electroencephalogram signals, IEEE Sensors Journal 18. doi:10.1109/JSEN.2018.2869775.

[43] Adnane, M., Jiang, Z. and Yan, Z. Sleep-wake stages classification and sleep efficiency estimation using single-lead electrocardiogram, Expert Systems with Applications: An International Journal 39 (2012) 1401-1413. doi:10.1016/j.eswa.2011.08.022.

[44] Redmond, S. and Heneghan, C. Electrocardiogram-based automatic sleep staging in sleep disordered breathing, Computers in cardiology. IEEE 30 (2003) 609-612.

[45] Hayet, W. and Yacoub, S. Sleep-wake stages classification based on heart rate variability, Biomedical Engineering and Informatics (BMEI), 5th International Conference (2012) 996999. doi:10.1109/BMEI.2012.6513040.

[46] Werteni, H., Yacoub, S. and Ellouze, N. Classification of sleep stages based on EEG signals, International Review on Computers and Software (IRECOS) 10 (2015) 174.

[47] An, P., at. al. A novel eeg sleep staging method for wearable devices based on amplitude-time mapping, 2019 IEEE 4th International Conference on Advanced Robotics and Mechatronics (ICARM) , 124-129 (2019).

[48] Zhang, J., Yao, R., Ge, W. and Gao.J. Orthogonal convolutional neural networks for automatic sleep stage classification based on single-channel EEG, Computer Methods and Programs in Biomedicine 183 (2020) 105089. doi:https://doi.org/10.1016/j.cmpb.2019.105089.

[49] Prucnal, M., and Polak, A. Effect of feature extraction on automatic sleep stage classification by artificial neural network, Metrol Meas Syst 24(2):229-40

[50] Hasan, P., and Mehmet, S. O. Epileptic seizure detection from EEG signals by using wavelet and Hilbert transform, MEMSTECH 2016 7(4). 
13th Joint Conference on Mathematics and Computer Science, October 1 - 3, 2020, ELTE, Hungary15

[51] Runarsson, T. and Sigurdsson, S. On-line Detection of Patient Specific Neonatal Seizures using Support Vector Machines and Half-Wave Attribute Histograms., (CIMCA-IAWTIC'06) 2 (2005): 673-677. 
Figures

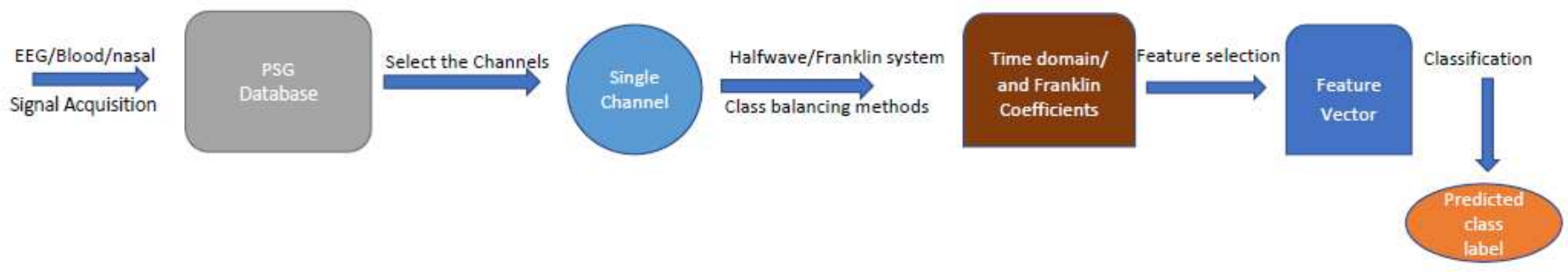

\section{Figure 1}

Framework of proposed method 